\title{
Research on Life Prediction of Train Control Center Based on Random and Fuzzy Theory
}

\author{
Xiao Chun $\mathrm{Wu}^{1}$ and Jian $\mathrm{Wu}^{2}$ \\ ${ }^{1,2}$ School of Automation \& Electrical Engineering, Lanzhou Jiaotong University, \\ Lanzhou, 730070, China \\ 1369038806@qq.com, ${ }^{2}$ wujian0915@126.com
}

\begin{abstract}
The health state assessment and remaining life prediction of Train Control Center (TCC) is an important premise to carry out state repair of equipment, which can effectively protect the safe operation of the system. The paper aims to assess the health state of the equipment by adopting the health index model of TCC based on fuzzy comprehensive evaluation. As to various uncertain factors the TCC has such as the operating environment, the quality of the equipment and maintenance, the life prediction model of TCC is established based on the evaluative result, namely health index HI. Further variation that the equipment health index changes with time is established to realize the life prediction of TCC. The example shows that the life of TCC can be predicted effectively by means of the health state assessment for the equipment. The railway department can obtain the corresponding data, which can provide the basis of the maintenance and update for the equipment.
\end{abstract}

Key words: Train Control Center; Health index; Life prediction; Random fuzzy theory

\section{Introduction}

In recent years, with the improvement of line speed and construction of High Speed Railway, CTCS-2 and CTCS-3 (CTCS, Chinese Train Control System) have been widely spread and applied. TCC (Train Control Center) is the core element of ground equipment, which plays an important role in ensuring the safety of train operation. "Maintenance and management rules for CTCS-2" issued by China Railway Corporation (formerly the Ministry of Railways) in 2007, clearly stipulates: the train control system equipment and facilities should be subject to the administration of life. Those that have reached the life cycle should not continue to use ${ }^{[1]}$. At present, railway equipment renewal is based on the running time. As the core element of CTCS, TCC life prediction has practical significance that it can provide theoretical foundation for the maintenance and update of equipment.

TCC is a complex structure and critical safe equipment. Secure host unit, as the core part of TCC, is responsible for logic processing and system managing task ${ }^{[2]}$. Only the failure of secure host unit can cause TCC to stop running. Therefore, the life prediction of TCC is the same as that of secure host unit. In recent years, there are many researches on safety assessment and failure diagnosis technology of CTCS. Reference [3] expounds hazard identification of TCC function applying FMEA (Failure Mode and Effects Analysis). Reference [4] analyses TCC security through data analysis platform. Reference [5] discusses diagnosis technology by means of Linux and Qt development library. It can be seen that TCC researches focus on safety analysis and fault diagnosis technology. However, with the development of railway information, computer technological application requires that it is necessary to analyze and forecast the real-time state of TCC. Researches on life prediction and real-time state assessment have been applied in the field of aerospace and electric power system, etc. ${ }^{[6-8]}$. Reference [9] carries out life analysis of transmitter and 
receiver in ZPW-2000A, which describes corresponding lifetime distribution, but it doesn't analyze real-time state. Therefore, researches on TCC health analysis and life prediction are lack of corresponding theoretical guidance. The paper establishes life prediction model of TCC, including life prediction model and health assessment model. Life prediction can obtain the remaining life of equipment, and that the change of equipment real time state is reflected by health index. It can achieve real-time analysis of the state and performance of TCC.

\section{Health Index Model of TCC}

The factors that affect the health state of TCC are numerous, complex and fuzzy. So the paper applies health index evaluative model of TCC based on fuzzy comprehensive evaluative method. The result that quantifies health state of TCC is defined as HI (health index). HI is an index, which reflects health degree of TCC. Regulation: the HI value ranges from 0 to 100 . The closer HI approaches 100, the higher TCC is to health level, on the contrary, the closer HI approaches 0, the lower TCC is to health level. HI=60 is defined as the critical point, which indicates that TCC has reached the life cycle, and the equipment should stop running for replacement. HI $>60$ represents system normal, HI $<60$ represents system failure ${ }^{[10]}$. The relationship between the health index and the health state evaluative level of TCC is shown in Table 1.

Table 1. Health state level of TCC

\begin{tabular}{|c|c|c|c|}
\hline Grade & HI Range & Health level & The description of health state \\
\hline I & $85 \leq \mathrm{HI} \leq 100$ & Health & Equipment parameters maintain stability \\
\hline II & $70 \leq \mathrm{HI} \leq 85$ & Sub-health & Equipment parameters appear deviation \\
\hline III & $60 \leq \mathrm{HI} \leq 70$ & Equipment exception & Equipment safety is lower than the standard \\
\hline IV & $0 \leq \mathrm{HI} \leq 60$ & Equipment failure & Equipment operation is failure \\
\hline
\end{tabular}

The steps of establishing the model of the TCC in health index are as follows:

(1) Determine the health indicators system $\boldsymbol{U}$

As shown in Figure 1, the paper establishes a collection of health state assessment factors $\boldsymbol{U}=\left\{u_{1}, u_{2}, u_{3}, u_{4}, u_{5}, u_{6}\right\}, u_{i}$ represents 6 kinds of influencing factors, which affects health state of evaluative object.

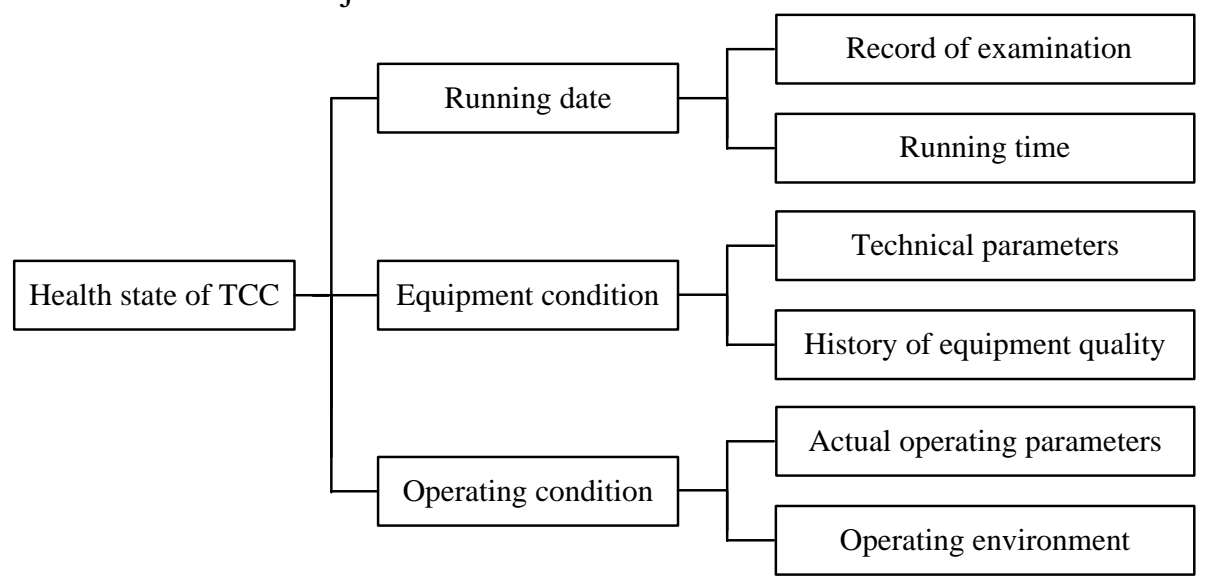

Figure 1. Health Index System of TCC 
(2) Establish evaluative set $\boldsymbol{V}$

$V_{i}$ represents the affecting factors on the health state of TCC. Due to the fact that the health state of TCC is a fuzzy concept, the health degree is divided into four grades (health, sub-health, exception and failure) according to the operating characteristics of TCC. It can be expressed as $\boldsymbol{V}=\left\{v_{1}, v_{2}, v_{3}, v_{4}\right\}$, as $v_{j}(j=1,2,3,4)$ is marked the $\mathrm{j}$-th evaluative level. According to the division of health state in table $1, V=\{100,85,70,60\}$ can be obtained.

(3) Set up weight set $\boldsymbol{A}$

After establishing index system, the paper will judge the weight of each index, and determine the corresponding weight of each index by means of analytic hierarchy process.

(4) Define the single factor evaluative matrix $\boldsymbol{R}$

If the $\mathrm{i}$-th factor is evaluative content for TCC in $\boldsymbol{U}$ and the $\mathrm{r}_{\mathrm{ij}}$ is membership degree for the $\mathrm{j}$-th level in $\boldsymbol{V}$, the fuzzy set $\boldsymbol{R}_{i j}=\left\{r_{i 1}, r_{i 2}, r_{i 3}, r_{i 4}, r_{i 5}, r_{i 6}\right\}$ expresses evaluative results. Therefore, it is necessary to determine the membership degree of the evaluative factors. For quantitative index, the concept of relative deteriorative degree is introduced, and then the membership function of each index under different conditions is established using the distributive function of the triangle. For qualitative index, the membership function of the index is determined by using the expert scoring method and fuzzy statistical method.

(5) Determine the fuzzy comprehensive evaluative model

The fuzzy evaluation of single factor reflects the influence for single factor affecting the evaluative object, but for those of multiple-factors, synthesizing single factor evaluative results are needed. Therefore, the fuzzy comprehensive evaluative matrix of TCC in health state is shown in the formula (1).

$$
B=A \& R=\left(a_{1}, a_{2}, \cdots, a_{6}\right)\left[\begin{array}{cccc}
r_{11} & r_{12} & \cdots & r_{16} \\
r_{21} & r_{22} & \cdots & r_{26} \\
\vdots & \vdots & & \vdots \\
r_{61} & r_{62} & \cdots & r_{66}
\end{array}\right]=\left(b_{1}, b_{2}, \cdots, b_{4}\right)
$$

(6) Synthesize evaluative results HI

$H I=\sum_{j=4}^{4} c_{j} v_{j}$

In Formula (2), the $v_{j}$ is element of the evaluative set $\mathrm{V}$, the meaning of $c_{j}$ is as follows: $c_{j}=b_{j} / \sum_{j=1}^{4} b_{j}$

In Formula (3), the $b_{j}$ is element of the matrix B in Formula (1).

\section{Remaining Life Prediction Model of TCC}

The core element of TCC is secure host unit, which belongs to electronic equipment. When TCC is running, it will be affected by various uncertain factors from the outside world, such as operation condition, the quality of equipment, monitoring and maintenance and so on. Because of those factors with random and fuzziness, the paper establishes the life prediction model of equipment by random fuzzy theory.

\subsection{Introduction of Random Fuzzy Theory}

Random fuzzy theory researches uncertain phenomena with random and fuzziness. In 2004, Liu Baoding put forward the definition of random variable, operator of expected value, key value and opportunity distribution and established complete random fuzzy theory on the basis of the theory of probability and trust, which provides a theoretical basis for dealing with random fuzzy variable ${ }^{[11]}$. The random fuzzy variable deals with 
random fuzzy event, which is a function of the collection that the credibility space mappings to the random variable, and it is defined as follows:

Definition 1 , If $\xi$ is a function that the credibility space $(\Theta, p(\Theta)$, Qr) mappings to the random variable collection, then the $\xi$ is a random fuzzy variable.

Definition 2, Let $\eta$ be a random variable defined on the probability space $(\Omega, \wedge, \operatorname{Pr}) . \delta$ is a fuzzy variable defined in the credibility space $(\Theta, \mathrm{p}(\Theta), \mathrm{Qr})$, then $\xi=\eta+\delta$ is a random fuzzy variable.

Definition 3, Let $\xi$ be a random fuzzy variable, and $\alpha, \beta \in(0,1]$, then:

$$
\begin{aligned}
& \xi_{\text {sup }}(\alpha, \beta)=\sup \{i \mid \mathrm{Ch}\{\xi \geq i\}(\alpha) \geq \beta\} \\
& \xi_{\text {inf }}(\alpha, \beta)=\inf \{i \mid \operatorname{Ch}\{\xi \leq i\}(\alpha) \geq \beta\}
\end{aligned}
$$

Where, the formula (4) represents the positive value of $\xi$ in $(\alpha, \beta)$, the Formula (5) indicates the pessimistic value of $\xi$ in $(\alpha, \beta)$.

Definition 4 , Let $\xi$ be a random fuzzy variable that is defined on the credibility space $(\Theta, \mathrm{p}(\Theta), \mathrm{Qr})$, And $A$ is the Borel set in $\mathrm{I}$, then the random fuzzy event $\xi \in \boldsymbol{A}$ is defined as a function of the type $(6)$ from the interval $(0,1]$ to $[0,1]$.

$$
\operatorname{Ch}\{\xi \in A\}(\alpha)=\sup _{\operatorname{Cr}(M) \geq \alpha} \inf _{\theta \in M} \operatorname{Pr}\{\xi(\theta) \in A\}
$$

\subsection{Life Characteristic Curve of TCC}

As the running time increases, the overall performance index of the TCC will decrease with the aging of the equipment until the equipment becomes failure. In Figure 2, the $\mathrm{P}-\mathrm{F}^{[12]}$ curve describes the deteriorative process of the device in health state. As the diagram of curves show that: from 0 to T1, the performance of the device is stable. From $\mathrm{T} 1$ to $\mathrm{T} 2$, the performance of the device decreases obviously, and the failed rate increases. After the time of T2, the performance index of the device trends to fall quickly, ultimately it is less than the technical standards until the equipment lose efficacy.

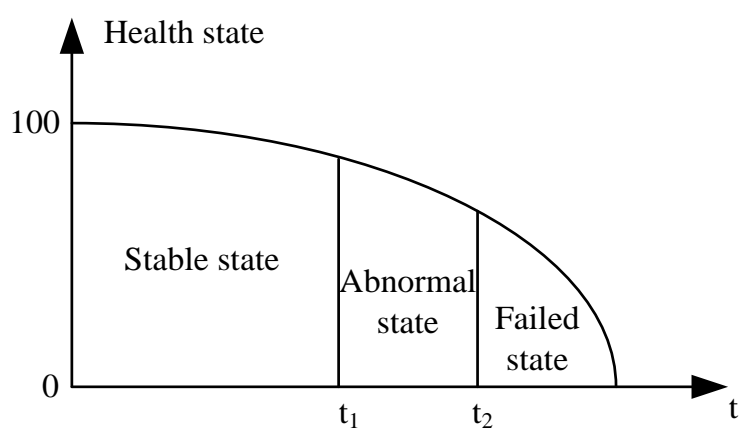

Figure 2. P-F curve

Figure 2P-F curve reflects the change of the equipment in health state. From Figure 2, it can be seen that the health state of the equipment is in accordance with exponential law.

\subsection{Determination of Random Fuzzy Distributive Function for Health Index}

According to the monitoring data of the microcomputer monitoring system, the record of the production and maintenance of TCC, the health index over the years of TCC is obtained by using the fuzzy comprehensive evaluative model. From the data, it can be known that the health state of TCC is decreasing with exponential law. Therefore, the 
random fuzzy distribution can describe accurately the distributive law of TCC in health index. According to the calculation of the health index, the exponential distributive function of TCC in health index is obtained by applying MATLAB least square fitting method. The solid line in Figure 3 shows that the distribution of TCC in health index is in line with the P-F curve.

Using curve fitting method can obtain the health index distributive function, as shown in Formula (7).

$$
H I(t)=a_{0}+b e^{c t}
$$

Where, $a, b, c$ are constants.

According to the curve $H I(t)=a_{0}+b e^{c t}$, shifting it can obtain the curves $H I(t)=a_{1}+b e^{c t}$ and $H I(t)=a_{2}+b e^{c t}$, as the dotted lines show in figure (3). And then, the parameter $a_{0}$ of the distributive function of the health index is represented by triangular fuzzy variable $a=\left(a_{1}, a_{0}, a_{2}\right)$. Therefore, the relationship between the health index and the running time of the TCC are represented by the random fuzzy distributive function, as is shown in the Formula (8).

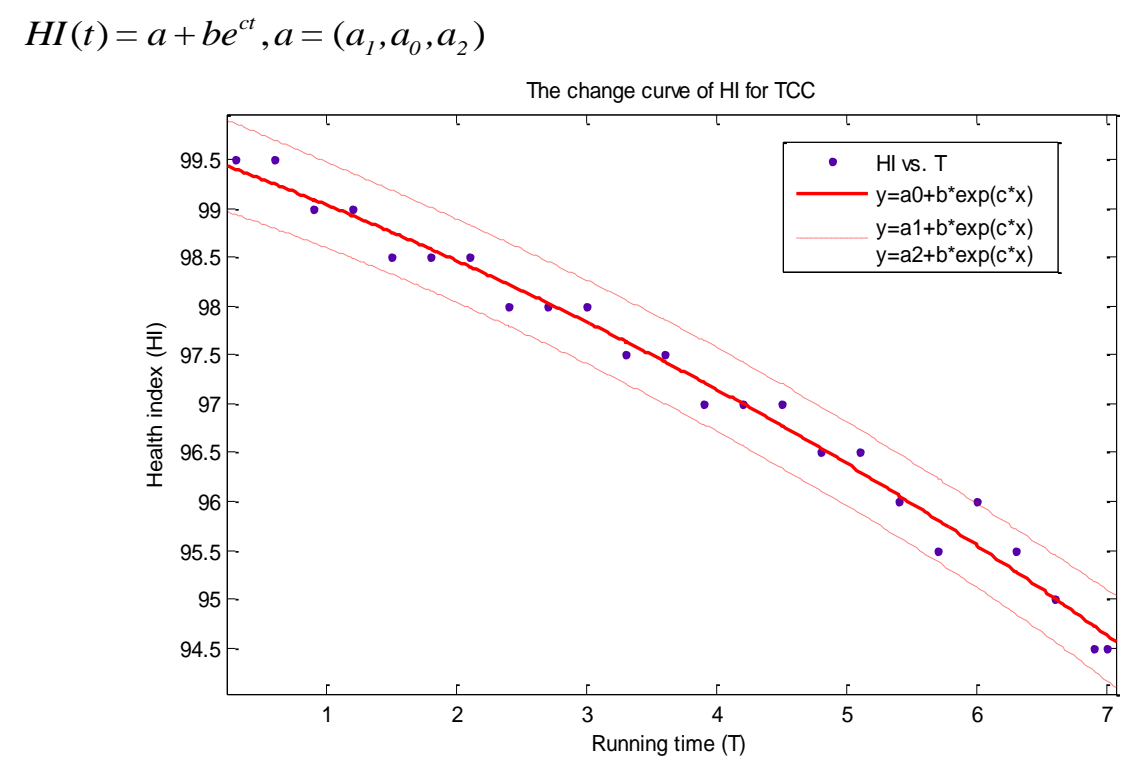

Figure 3. The Changing Ccurve of $\mathrm{HI}$ for TCC

\subsection{Establishment of Life Prediction Model based on Random Fuzzy Theory}

The paper predicts the life of TCC according to health index. The life of TCC is the time of normal work until the health index is reduced to the minimum allowable value during the operation. The time is shown in Formula (9).

$$
T=\max \left\{t \mid H I(t) \geq H I_{L}\right\}
$$

In the Formula (9), $\mathrm{HI}_{\mathrm{L}}$ represents the lowest health index that is allowed during the operation of TCC.

According to the Formula (8) and (9), the Formula (10) can be got:

$$
a+b e^{c t} \geq H I_{L}
$$

As the health state curve shows exponential decline, so there is $b<0, c>0$, the Formula (10) can be transformed to Formula (11). 


$$
e^{c t} \leq \frac{\left(H I_{L}-a\right)}{b}
$$

According to logarithmic transformation of the Formula (11), the formula (12) can be got as follows:

$t \leq \frac{1}{c} \ln \left(\frac{\left(H I_{L}-a\right)}{b}\right)$

In Formula (12), $H I_{L}, b, c$ are constants, and $a$ is a random fuzzy variable, therefore, the right side of inequality (12) is a random fuzzy variable. Consequently, in the given confidence level of $\alpha$ and $\beta$, the optimistic value of random fuzzy variable $\bar{t}$ is the maximum value of $T$ in the formula (9), then the $\bar{t}$ is shown in Formula (13).

$\operatorname{Ch}\left\{\frac{1}{c} \ln \left(\frac{\left(H I_{L}-a\right)}{b}\right) \geq \bar{t}\right\}(\alpha) \geq \beta$

The formula (13) is the life prediction model of TCC based on random fuzzy theory. The $T=\bar{t}$ named the life of TCC can be obtained through model solution, then the remaining life of TCC can be expressed as $T_{t}=T-t$.

\subsection{Solution of Life Prediction Model}

Aiming at the solution of random fuzzy variable, Liu put forward the random fuzzy simulative technique ${ }^{[13]}$, which can solve random fuzzy variable. In order to solve random fuzzy variable $T$ of the life prediction model for TCC, the paper establishes algorithm flow based on random fuzzy simulative technique, the steps of algorithm flow are as follows:

Step 1: $\operatorname{Set} T=0$;

Step 2: The sample $\theta_{k}$, which is $\operatorname{met} \operatorname{Cr}\left\{\theta_{k}\right\} \geq \varepsilon / 2(\mathrm{k}=1,2, \cdots, N)$, can be generated uniformly from the credibility space $\Theta$.Where, $\varepsilon$ is small enough of positive number, and $v_{k}=\left(2 \operatorname{Cr}\left\{\theta_{k}\right\}\right) \wedge 1$ is set.

Step 3: For any one $\theta_{k}$, the maximum value of $f\left(\theta_{k}\right)$ can be obtained, which is met

$$
\operatorname{Pr}\left\{f\left(\xi\left(\theta_{k}\right)\right) \geq f\left(\theta_{k}\right)\right\} \geq \beta \text {. }
$$

Step 4: The $r$ is solved, where, $\mathrm{L}(\mathrm{r})=\frac{1}{2}\left(\max _{1 \leq k \leq N}\left\{v_{k} \mid f\left(\theta_{k}\right) \geq r\right\}+\min _{1 \leq k \leq N}\left\{1-v_{k} \mid f\left(\theta_{k}\right)<r\right\}\right)$.

Step 5: If $r>T$, then set $T \leftarrow r$, and repeat step 2 to step $5 \mathrm{~N}$ times. If $r<T$, then repeat step 2 to step $5 \mathrm{~N}$ times.

Step 6: output life $T$.

Therefore, the algorithm flowchart of the life prediction model for TCC is shown in Figure 4. 


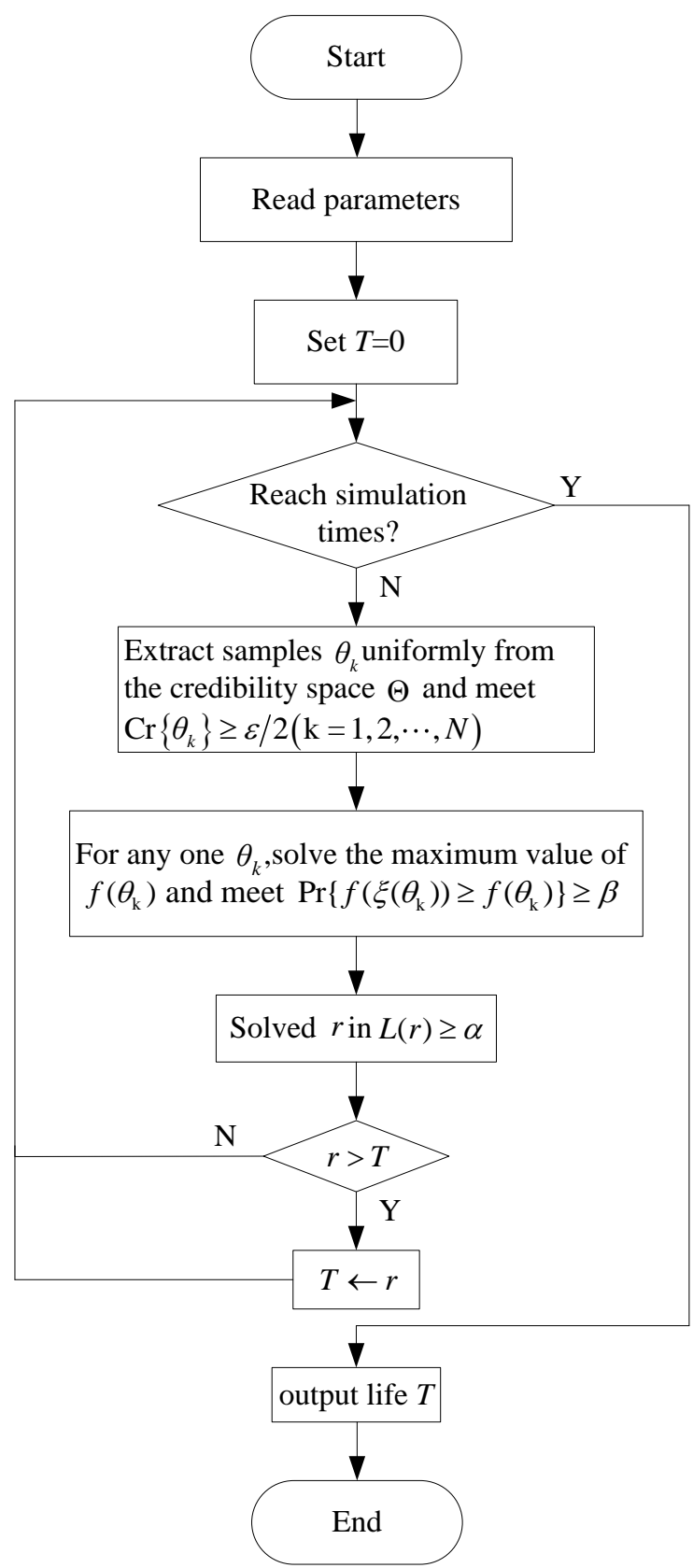

Figure 4. Algorithm Flowchart of the Life Prediction Model for TCC

\section{Case Verification and Result Analysis}

Taking a 7-year TCC of a railway administration as an example, the paper calculates the remaining life and evaluates the health status to verify the rationality and practicality of the above method.

According to the field data and technical conditions of TCC, the health state assessment model is used to calculate the health index of TCC.

(1) Determine the weight of each index 
The weight of each index is analyzed and calculated by applying analytic hierarchy process, and the results are shown in Table 2.

Table 2. Index Weight

\begin{tabular}{|c|c|c|c|}
\hline Evaluative Index & Weight & Evaluative Index & Weight \\
\hline Record of examination & 0.245 & Running time & 0.120 \\
\hline Technical parameters & 0.150 & History of equipment quality & 0.037 \\
\hline Actual operating parameters & 0.338 & Operating environment & 0.110 \\
\hline
\end{tabular}

(2) Determine the index score

According to the actual operating condition of TCC, the fuzzy evaluative model of the health state for TCC and the deteriorative process of TCC, the expert scoring method is applied to calculate the score of each index, and the results are shown in Table 3.

Table 3. Index Score

\begin{tabular}{|c|c|c|c|}
\hline Evaluative Index & Score & Evaluative Index & Score \\
\hline Record of examination & 96 & Running time & 94 \\
\hline Technical parameters & 95 & History of equipment quality & 96 \\
\hline Actual operating parameters & 94 & Operating environment & 93 \\
\hline
\end{tabular}

(3) Calculate the health index of TCC

According to the evaluative index weight of Table 2 and the evaluative index score of Table 3, formula 2 is applied to calculate the health index of TCC:

$$
\mathrm{HI}=0.245 \times 96+0.120 \times 94+0.150 \times 95+0.037 \times 96+0.338 \times 94+0.110 \times 93=94.604
$$

The health index of TCC is 94.5 (data archiving facilitates statistics and calculation). In the same way, the paper calculates the health index over the years of TCC. The results are shown in Table 4.

Table 4. Health Index of Past Years for TCC

\begin{tabular}{|c|c|c|c|c|c|}
\hline Operating years & HI & Operating years & HI & Operating years & HI \\
\hline 0.5 & 99.5 & 3 & 98 & 5.5 & 96 \\
\hline 1 & 99 & 3.5 & 97.5 & 6 & 95.5 \\
\hline 1.5 & 98.5 & 4 & 97 & 6.5 & 95.5 \\
\hline 2 & 99 & 4.5 & 97 & 7 & 94.5 \\
\hline 2.5 & 98.5 & 5 & 96.5 & - & - \\
\hline
\end{tabular}

From the changing curve of TCC in Figure 3, the health index and running time of TCC present distributive law of the index function. According to the health index of TCC in Table 4, the distributive function of health index for TCC can be obtained by using the MATLAB least squares fitting method. The results are shown in Figure 5. 


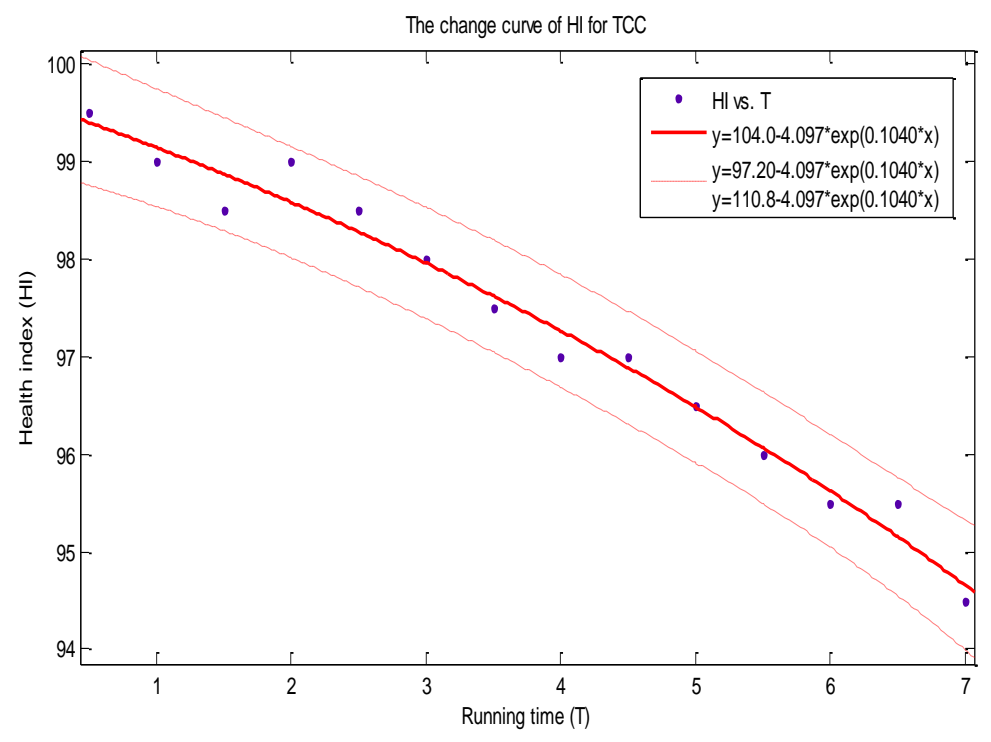

Figure 5. The Changing curve of HI for TCC

According to Figure 5, the distributive function of $\mathrm{HI}$ for TCC is $f(t)=104.0-4.097 e^{0.1040 t}$, just as the solid line shows in Figure 5. The other two dividing lines of $\mathrm{HI}$ distributive functions $f(t)=97.20-4.097 e^{0.1040 t}$ and $f(t)=110.8-4.097 e^{0.1040 t}$ can be got by vertical shifting, as the dotted lines show in Figure 5. Comprehensively, the expression of the random fuzzy distributive function of TCC is shown in the Formula (14).

$f(t)=a-4.097 e^{0.1040 t}$

Where, $a=(97 \cdot 2,104 \cdot 0,110.8)$.

The result of formula (14), which is the random fuzzy distributive function, substitutes into formula (13), the formula (15) can be obtained.

$\operatorname{Ch}\left\{\frac{1}{0.1040} \ln \left(\frac{H I_{L}-a}{-4.097} \geq \bar{t}\right)\right\}(\alpha) \geq \beta$

The optimistic value $\bar{t}$ in formula (15) is the life $T$ of TCC, which is to be solved. Under the condition of the lowest health index is 60 and the confidence level is 0.92 , namely $\alpha=60, \beta=0.92$, applying random fuzzy simulative technique solves the life prediction model of TCC, and it can be obtained that $\bar{t}=22.72$, as a result, the maximum life of TCC is $T_{\max }=22.72$ years. Then the remaining life is $T_{t}=22.72-7=15.72$ years. Similarly, when the minimum health index is 85 and the confidence level is 0.92 , namely $\alpha=85, \beta=0.92$, the health life of TCC is $T_{H L}=14.56$ years. Therefore, TCC to maintain the health state of operating time is 14.56 years, and the remaining health life is $T_{h}=14.56-7=7.56$ years.

\section{Conclusion}

The paper establishes the assessment model of TCC in health index based on fuzzy comprehensive evaluation and calculates the health index of past years for TCC through the model. Meanwhile, the paper establishes the life prediction model of TCC based on random fuzzy theory, and the changing law of health state for TCC can be reflected through health index. In the case of the field running TCC, the paper calculates the 
maximum life $T_{\max }$ and the health life $T_{H L}$ by means of the life prediction model. The maximum life $T_{\max }$ can calculate the remaining life of TCC, and the health life $T_{H L}$ can reflect the holding time of health state for TCC. The calculative results can provide data for the maintenance and update of TCC, and provide the theoretical basis, which can achieve life management of CTCS.

\title{
References
}

[1] China Railway Corporation, "Maintenance and management rules for CTCS-2", Beijing: China Railway Publishing house, (2007).

[2] China Railway Corporation, "CTCS-2 grade train control system”, Beijing: China Railway Publishing house, (2013).

[3] Y. Z. Zhao, "Research on application of function safety analysis of train control center", Chengdu: Southwest Jiaotong University, (2009), pp.23-25.

[4] P. Wang, "Data communication and security study of train control center", Chengdu: Southwest Jiaotong University, (2014), pp.37-40.

[5] J. P. Li, "Research on Fault Diagnosis Technology in TCC of Passenger Dedicated Railway", Beijing: Beijing Jiaotong University, (2010), pp.5-12.

[6] R. Li, B. Han and Y. Lu, "Transformer's economic life assessment based on random and fuzzy theory", Power System Protection and Control, vol.42, no.1, (2014), pp.9-16.

[7] S. H. Ren and H. F. Zuo, "A Combined Prediction Method for the Residual-life of Civil Aviation Engines Based on Performance Degradation", Mechanical Science and Technology for Aerospace Engineering, vol.30, no.1, (2011), pp.23-29.

[8] X. G. Li and Y. Chang, "Remnant Life Estimation of Power Transformer Based on Health Index", High Voltage Apparatus, vol.48, no.12, (2012), pp.80-85.

[9] F. Zuo, "The life data analysis of the transmitter and receive of ZPW-2000A system", Chengdu: Southwest Jiaotong University Master Degree Thesis, (2011), pp.23-30.

[10] R. Li, H. L. Liu and Y. Lu, "A combination method for distribution transformer life prediction based on cross entropy theory", Power System Protection and Control, vol.42, no.2, (2014), pp.97-103.

[11] B. D. Liu and J. Peng, "Course of uncertainty theory", Beijing: Tsinghua University press, (2005).

[12] X. H. Meng, Y. T. Zhou and W. Wang, "A study on the prediction model of remaining life equipment based on exponential distribution", China Ship repair, vol.25, no.5, (2012), pp.42-44.

[13] K. Huang, "Study on the evaluation of residual service life for high voltage circuit breaker", Beijing: North China Electric Power University, (2013), pp.27-30.

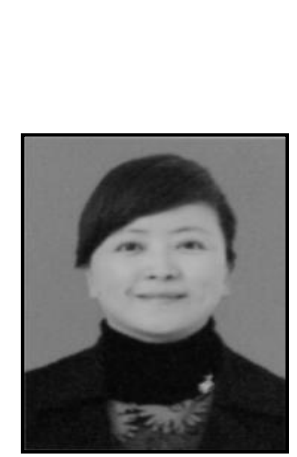

\begin{abstract}
Authors
Xiaochun Wu, She received her master's degree in Traffic Information Engineering \& Control from Lanzhou Jiaotong University and she is a professor at Lanzhou Jiaotong University. She is currently researching on Chinese Train Control System and digital signal processing.
\end{abstract}

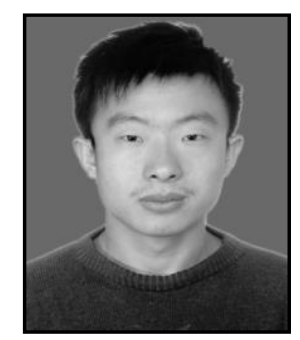

Jian $\mathbf{W u}, \mathrm{He}$ received the automatic control degree form Lanzhou Jiaotong University, now he is a master student in school of Automation \& electrical Engineering from Lanzhou Jiaotong University. His current research interests include risk evaluation of Chinese Train Control System. 